\title{
Transgenic pigs designed to express human $\alpha$-galactosidase to avoid humoral xenograft rejection
}

\author{
J. Zeyland • B. Gawrońska • W. Juzwa • J. Jura • \\ A. Nowak • R. Słomski • Z. Smorąg • M. Szalata • \\ A. Woźniak • D. Lipiński
}

Received: 24 September 2012 / Revised: 18 April 2013 / Accepted: 27 May 2013 / Published online: 19 June 2013

(C) The Author(s) 2013. This article is published with open access at Springerlink.com

\begin{abstract}
The use of animals as a source of organs and tissues for xenotransplantation can overcome the growing shortage of human organ donors. However, the presence of xenoreactive antibodies in humans directed against swine Gal antigen present on the surface of xenograft donor cells leads to the complement activation and immediate xenograft rejection as a consequence of hyperacute reaction. To prevent hyperacute rejection, it is possible to change the swine genome by a human gene modifying the set of donor's cell surface proteins. The gene construct pGal-GFPBsd containing the human gene encoding $\alpha$-galactosidase enzyme under the promoter of $E F-1 \alpha$ elongation factor ensuring systemic expression was introduced by microinjection into a male pronucleus of the fertilised porcine oocyte. As a result, the founder male pig was obtained with the transgene mapping to chromosome $11 \mathrm{p} 12$. The polymerase chain reaction (PCR) analysis revealed and the Southern analysis confirmed transgene integration
\end{abstract}

J. Zeyland $(\bowtie) \cdot$ B. Gawrońska $\cdot$ R. Słomski $\cdot$ M. Szalata

D. Lipiński

Department of Biochemistry and Biotechnology, Poznan

University of Life Sciences, Dojazd 11, 60-632 Poznan, Poland

e-mail: jzeyland@yahoo.com

A. Nowak $\cdot$ R. Słomski $\cdot$ M. Szalata $\cdot$ D. Lipiński

Institute of Human Genetics, Polish Academy of Sciences,

Strzeszynska 32, 60-479 Poznan, Poland

W. Juzwa

Department of Biotechnology and Food Microbiology, Poznan University of Life Sciences, Wojska Polskiego 48, 60-627 Poznan, Poland

J. Jura $\cdot$ Z. Smorag

Department of Animal Reproduction, National Research Institute of Animal Production, 32-083 Balice, Poland

A. Woźniak

The NanoBioMedical Centre, Umultowska 85, 61-614 Poznan, Poland estimating the approximate number of transgene copies as 16. Flow cytometry analysis revealed a reduction in the level of epitope Gal on the cell surface of cells isolated from F0 and F1 transgenic animals. The complement-mediated cytotoxicity assay showed increased viability of the transgenic cells in comparison with the wild-type, which confirmed the protective influence of $\alpha$-galactosidase expression.

Keywords Epitopes $\cdot$ Transgenic pigs $\cdot$ Xenotransplantation

\section{Introduction}

Decreasing the frequency of chronic graft rejections, decreasing the toxicity of immunosuppressive treatments, inducing tolerance, and ensuring a sufficient number of graft organs are currently the most important challenges of modern transplantology. While the number of people awaiting transplantation is increasing every year, the availability of organs for transplantation is limited. In 2011, according to the Organ Procurement and Transplantation Network (OPTN), the number of registered donors in the United States of America was 11,713 (including living donors, which constituted $42.12 \%$ ), and the number of patients awaiting transplantation was 112,728 . There was a total demand for 122,438 organs, while the number of transplantations was only 23,745 . For example, 16,857 patients awaited liver transplantation, while the available organs could cover only $34.34 \%$ of the demand. In fact, in 2011, 5,288 liver transplantations were conducted in $31 \%$ of awaiting patients (OPTN 2011). The introduction of improvements in the system of organ registration and distribution and better general health of the society obtained through promoting healthy lifestyle are not enough to improve the short supply of potential organs and transplantation tissues. This situation resulted in increased interest in studies on alternative sources of organs. Transplantation 
involving the use of organs from other mammals as human transplants are called xenotransplantation. They have become the most obvious strategy of solving the problem and a chance to save lives.

From both the genetic and physiological points of view, the phylogenetic proximity of apes and humans is the strongest argument standing for xenotransplantations. However, it is also one of the biggest obstacles, involving the risk of infections, particularly with animal viruses. Due to the large phylogenetic distance and similar anatomical and physiological parameters, especially in respiratory and circulatory systems, choosing pigs as a donor of tissues and organs for transplantation seems to be justifiable. The size diversity between different types of pigs allows for choosing organs of size adjusted to different recipients. Pigs, as an easybreeding species, can be housed under strict barrier conditions and screened for pathogenic xenozoonosis at regular intervals (Cooper 2012). However, it should be expected that the large phylogenetic distance will be the reason for a number of immunological complications after pig-to-human transplantations. Binding the naturally occurring preformed antibodies of recipient to Gal $\alpha(1,3) \mathrm{Gal}$ epitopes on the endothelial surface makes apes reject grafts from pigs by means of hyperacute rejection (HAR). The Gal $\alpha(1,3) \mathrm{Gal}$ epitope is formed by connecting a molecule of galactose to $\mathrm{N}$ acetyllactosamine by the 1,3-glycosidic bond in the presence of $\alpha 1,3$-galactosyltransferase (GGTA1, $\alpha 1,3 \mathrm{GT}$ ) (Sandrin and McKenzie 1994). Both the enzyme and the sugar residue are not present in humans and apes of the Old World (Galili et al. 1988). It is suggested that, in ancestors of order primates, the gene coding for 1,3-galactosyltransferase was inactivated (Galili and Swanson 1991). Humans have, in turn, xenoreactive antibodies (XNA), which recognise the Gal $(1,3)$ Gal epitope present on several dozens of molecules on the surface of endothelial cells in the pig and activate the enzymatic cascade of the complement system responsible for rapid graft rejection. Among all xenoreactive antibodies in the human organism, even $90 \%$ recognise the $\mathrm{Gal} \alpha(1,3) \mathrm{Gal}$ epitope. Anti-Gal antibodies constitute approximately $1-2.4 \%$ of IgG and $1-8 \%$ of IgM immunoglobules circulating in human blood (Sandrin et al. 1993; McMorrow et al. 1997). One of the factors of preventing the rejection of xenografts is to develop methods of controlling or limiting the hyperacute rejection of the immunological system through, inter alia, decreasing the amount of Gal $\alpha(1,3)$ Gal epitopes on the surface of donor cells. This problem can be overcome by the development of pigs homozygous for $\alpha 1$,3-galactosyltransferase gene-knockout (GTKO). If dominant pig carbohydrate and glycoprotein epitopes other than Gal $\alpha(1,3)$ Gal are shown to be physiologically pivotal in the pig-to-human combination, additional gene knockouts could also be envisioned (Pierson 2009). Another strategy is to use digestion to remove terminal residues of epitope D-galactose from the surface of pig cells by specific $\alpha$-galactosidase (GLA)
(Luo et al. 1999). The use of GLA isolated from coffee seeds decreased the cytotoxic activity of natural antibodies present in both human serum and crab-eating macaque by $59-90 \%$ in relation to endothelial cells of the pig (SEC) (LaVecchio et al. 1995). The perfusion of organs with GLA requires isolating and purifying significant amounts of enzyme and maintaining optimal conditions for its activity, which, in consequence, generates costs, making it hardly usable on a broader scale (Ogawa et al. 2000). Vast progress in obtaining transgenic livestock resulted in the possibility of remodelling the genome of the pig for xenotransplantations through the introduction of gene coding for human $\alpha$-galactosidase. The expression of human GLA was obtained in studies on pig cell lines. The result was a 30-fold decrease in the level of $\mathrm{Gal} \alpha(1,3) \mathrm{Gal}$ epitope on the endothelial surface (Osman et al. 1997). A reduction in the amount of $\operatorname{Gal} \alpha(1,3) \mathrm{Gal}$ epitope was observed in similar studies with the use of COS cells and NIH 3T3 (Yan et al. 2003); however, $\alpha$-galactosidase does not remove the residues of D-galactose from the Gal antigen entirely. At the same time, it is suggested that, in the case of isolated enzymes, GLA and $\alpha 1,2$-fucosyltransferase $(\alpha 1,2 \mathrm{FT})$ coexpression is used. The enzyme $\alpha 1,2$-fucosyltransferase competes with $\alpha 1,3$-galactosyltransferase, which is responsible for the synthesis of $\operatorname{Gal} \alpha(1,3) \mathrm{Gal}$ epitope, for the same substrate, $\mathrm{N}$ acetyllactosamine (N-lac), catalysing the addition of fucose, which results in the formation of the so-called $\mathrm{H}$ structure, commonly present in mammals (Larsen et al. 1990; Sandrin et al. 1995). The additive effect of both enzymes resulting in the total elimination of Gal antigen expression on the cell surface was observed in the case of cotransfection of different types of cells with genes coding for GLA and $\alpha 1,2 \mathrm{FT}$ (Osman et al. 1997; Yan et al. 2003; Jia et al. 2004).

The aim of this study was to prepare an expressive pGalGFPBsd gene construct containing the sequence coding for enzyme $\alpha$-human galactosidase controlled by the promoter of $E F-1 \alpha$ elongation factor ensuring the systemic expression of transgene and introducing it to the genome of pig by microinjection. In this study, we present the results characterising transgenic pigs with human $\alpha$-galactosidase expression using molecular, cytogenetic and functional approaches. The results are confronted with data obtained for TG1154 transgenic pig with $\alpha 1,2$-fucosyltransferase expression (Lipiński et al. 2010) in order to conduct cumulative transgenesis in the future for cumulating the activity of both enzymes in one organism for the needs of xenotransplantation.

\section{Materials and methods}

Gene construct

The pGal-GFPBsd gene construct was obtained by introducing cDNA coding for $\alpha$-galactosidase $(1,290 \mathrm{bp})$ into a 
pTracer-EF/Bsd A (5,987 bp) plasmid containing a strong, constitutive promoter of $1 \alpha$ subunit of human elongation factor $E F(\mathrm{hEF}-1 \alpha)(1,179 \mathrm{bp})$ and a poly(A) sequence of cattle GH gene (225 bp). Vector pTracer-EF/Bsd A expresses the blasticidin resistance gene as a fusion product with the Cycle 3 GFP, allowing the selection and non-invasive detection of recombinant proteins (Invitrogen). The coding sequence was amplified on single-strand cDNA template synthesised by oligo $(\mathrm{dT})_{20}$ primers and the SuperScript III kit (Invitrogen). Total RNA was isolated from human white blood cells from peripheral blood with the method based on guanidine isothiocyanate (Chomczynski and Sacchi 1987). Modified primers were used for amplification. Additionally, a sequence recognised by the EcoRI enzyme (5'-GCGAA TTCCATGCAGCTGAGGAACCCAGAACTACA-3') was placed in the $\mathrm{F}$ primer and a sequence recognised by the NotI enzyme (5'-GGCGGCCGCTTAAAGTAAGTCTTT TAATGACATCTGCA-3') was placed in the $\mathrm{R}$ primer. Polymerase chain reaction (PCR) of $25 \mu \mathrm{l}$ was conducted in the following conditions: $94{ }^{\circ} \mathrm{C}, 60 \mathrm{~s} ; 58^{\circ} \mathrm{C}, 60 \mathrm{~s} ; 72^{\circ} \mathrm{C}$, $90 \mathrm{~s} ; 35$ cycles. The reaction mixture contained $1 \mu \mathrm{l}$ of cDNA, $10 \mathrm{pM}$ of primers, $7.5 \mathrm{nM}$ dNTP, $1 \times$ buffer and $1 \mathrm{U}$ of DNA Taq polymerase (Sigma). PCR product was digested by EcoRI (Fermentas) and NotI (Fermentas), purified and ligated with the $\mathrm{p}$ Tracer-EF/Bsd A vector, which was digested with the same endonucleases. The product was sequenced using automated genetic analysers (Applied Biosystems Prism). The construction prepared for microinjection was purified by the PureLink ${ }^{\mathrm{TM}}$ HiPure Plasmid DNA Maxiprep Kit (Invitrogen) and then linearised with the Eam1105I enzyme.

\section{Microinjection}

Exogenous DNA as a pGal-GFPBsd gene construct was inserted into the male pronucleus of a fertilised egg cell. Microinjection was performed with the use of two micromanipulators under a reverse microscope with differential interference contrast. The applied magnification was objective $40 \times \mathrm{X}$ eyepiece $=450 \times$. DNA was injected into the male pronucleus with the use of an injector or a syringe connected to the holder for a manipulator. The volume of inserted DNA was 2-5 pl. DNA was inserted until the moment when the pronucleus increased its volume by $50 \%$. The DNA concentration was $2-4 \mathrm{ng} / \mu \mathrm{l}$. After the injection, the damaged zygotes were removed. Selected zygotes with the GFP signal detected were transplanted into the fallopian tubes of synchronised recipients.

Screening of transgene

Analysis included genomic DNA isolation from biopsies of potentially transgenic animals with the use of proteinase $\mathrm{K}$.
PCR was conducted on DNA template. Two pairs of primers were used for amplification. Both $\mathrm{F}$ primers were complementary to the $\mathrm{hEF}-1 \alpha$ promoter sequence, and both $\mathrm{R}$ primers were complementary to the sequence of $\alpha$-galactosidase (Fig. 1). A PCR product of $464 \mathrm{bp}$ is formed with the use of the first pair of $\mathrm{F} 1$ (5'-GGGGAGGGGTTTTATGCGATG GAG-3') and R1 (5'-CTGGCTCTTCCTGGCAGTCA-3') primers, whereas a second product of $861 \mathrm{bp}$ is formed with the use of the F2 (5'-ACCAGTTGCGTGAGCGGAAAG ATG-3') and R2 (5'-GGCGAATCCCATGAGGAAAG-3') primers. PCR was conducted in $20 \mu \mathrm{l}$ in the following conditions: $94{ }^{\circ} \mathrm{C}, 40 \mathrm{~s} ; 60{ }^{\circ} \mathrm{C}, 30 \mathrm{~s} ; 72{ }^{\circ} \mathrm{C}, 60 \mathrm{~s} ; 35$ cycles. The reaction contained $100 \mathrm{ng}$ of genomic DNA, $10 \mathrm{pM}$ of primers, $7.5 \mathrm{nM}$ of dNTP, $1 \times$ buffer and $1 \mathrm{U}$ of DNA Taq polymerase (Sigma).

\section{Southern hybridisation analysis}

For Southern analysis, total genomic DNA from the wildtype and analysed transgenic pig was extracted from white blood cells from peripheral blood with the method based on guanidine isothiocyanate. $5.6 \mu \mathrm{g}$ of each DNA was digested with restriction enzyme $E c o$ RI and hybridised with a probe of $464 \mathrm{bp}$. The probe was a 32P-dATP-labelled PCR product of $464 \mathrm{bp}$ formed on plasmid DNA (pGAL-GFPBsd) with the use of the first pair of primers (F1 and R1) used for the integration analysis (described above). The EcoRI enzyme digests the genomic DNA of pig as well as the pGALGFPBsd sequence (only one site). Digestion of the pGALGFPBsd sequence with EcoRI gives a fragment of $7.3 \mathrm{~kb}$ that is identical to the size of the introduced genetic construct. Additionally, for determining the transgene copy number, to the digested DNA of wild-type pig, known amounts of plasmid-containing transgene insert DNA was added. Then, DNA samples were electrophoresed using $1 \%$ agarose gel in $1 \times$ TBE, 40 V overnight. Denatured and neutralised DNA immobilised in dried gel was hybridised to a transgenespecific probe overnight $\left(55^{\circ} \mathrm{C}, 5 \times \mathrm{SSPE}, 0.1 \% \mathrm{SDS}\right)$.

Determination of the absolute number of transgene copies in transgenic pigs

Since $1 \mathrm{ng}$ of DNA corresponds to the molecular mass of $606,060,600 \mathrm{MDa}$ and the molecular mass of the swine genome amounts to $1,820,000 \mathrm{MDa}$, therefore, $1 \mathrm{ng}$ of swine genomic DNA contains approximately 333 copies of the genome. $5.6 \mu \mathrm{g}$ of swine genomic DNA sample per gel line were used, hence, each of the examined samples contained about $1,864,800$ copies of the swine genomic DNA. In order to calculate the number of copies of the transgene incorporated into a single genome of transgenic animals, the absolute number of copies of the transgene in titration samples (respectively, 124,122,350, 62,061,175, 31,030,587, 15,515,293, 


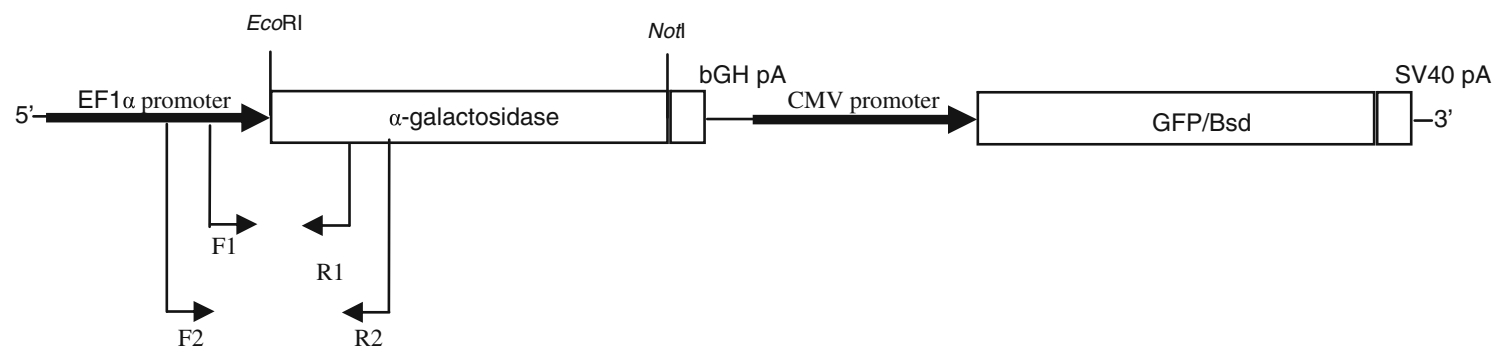

Fig. 1 The scheme shows the gene construct pGal-GFPBsd encompassing a strong, constitutive $E F-1 \alpha$ promoter $(1,179 \mathrm{bp}), \mathrm{cDNA}$ of the gene coding for human $\alpha$-galactosidase $(1,290 \mathrm{bp})$ and the poly(A) sequence of the cattle GH gene (225 bp). Moreover, the vector contains the sequence of Bsd gene coding for blasticidin antibiotic resistance joined with the GFP

$7,757,646$ copies) was divided by the number of copies of the genomic DNA. In the case of heterozygotic transgenic animals, the transgene occurs only on one of the pair of homologue chromosomes; therefore, in order to calculate the number of transgene copies built into one of the chromosomes, the result obtained from the calculations was multiplied by 2 .

\section{Skin fibroblasts isolation and cultivation}

The experimental material comprised skin fibroblasts obtained from the tissues of wild-type and transgenic pigs. Sterile tissues were rinsed in PBS solution containing antibiotics $(50 \mu \mathrm{g} / \mathrm{ml}$ gentamicin sulphate, $100 \mathrm{IU}$ penicillin and $50 \mu \mathrm{g} / \mathrm{ml}$ streptomycin). Skin fragments were cut and digested in $5 \mathrm{ml}$ trypsin-EDTA solution in PBS and then centrifuged. The cell sediment was suspended in enriched DMEM medium (20\% FBS, $1 \%$ antibiotic/antimycotic; Sigma). Culturing was carried out at the temperature of $37{ }^{\circ} \mathrm{C}$ at $5 \% \mathrm{CO}_{2}$ content. Transgenic cultures were checked for GFP signal. Cultures of full confluence were passaged to new $25 \mathrm{~cm}^{2}$ culture flasks.

\section{Mapping of the pGal-GFPBsd transgene}

Transgene mapping was conducted by fluorescent in situ hybridisation (FISH) with the use of in-vitro-cultivated cell lines. Pig karyotype evaluation was performed before conducting FISH. It involved the trypsinisation of chromosome preparations with trypsin and staining them with Giemsa stain solution. The obtained chromosome bands were analysed with the use of a microscope with a camera and appropriate software (Ikaros, MetaSystems). FISH was used for random primer hybridisation of the pGal-GFPBsd gene construct into the metaphase chromosomes obtained earlier. Hybridisation was conducted at $37{ }^{\circ} \mathrm{C}$ for $17 \mathrm{~h}$. Signal detection was performed with the use of antibodies against biotin and detected by fluorescein. In order to enable observation under a fluorescent microscope, DAPI/Antifade was used for dying. The image analysis was performed with sequence under the control of the CMV promoter. The scheme also shows the locations of the primers used for the screening of the transgene (F1 and $\mathrm{R} 1, \mathrm{~F} 2$ and R2) and the Southern probe binding site (between F1 and R1 primers)

the use of an Olympus 3X41 fluorescent microscope and the digital image analysis system MetaSystems 4000 ISIS Version 5.0. Transgene location was determined based on the standardised domestic pig karyotype (Gustavsson 1988).

\section{Flow cytometry}

Having reached full confluence, the skin fibroblasts cultivated in vitro were washed with Hanks solution and trypsinised ( $0.25 \%$ trypsin, $0.02 \%$ EDTA). Fibroblasts were collected by centrifugation. The cells were suspended and incubated in PBS containing lectin $(10 \mu \mathrm{g} / \mathrm{ml})$ and $5 \% \mathrm{FBS}$, at $4{ }^{\circ} \mathrm{C}$, for $45 \mathrm{~min}$. Lectin BS-IB4 isolated from Griffonia simplicifolia (Bandeiraea simplicifolia) conjugated with AlexaFluor 647 fluorochrome (Invitrogen) was used for the specific detection of Gal epitope. The flow cytometer (cell sorter) BD FACS Aria ${ }^{\mathrm{TM} I I I}$ (Becton Dickinson) with four lasers (375 nm, $405 \mathrm{~nm}, 488 \mathrm{~nm}$ and $633 \mathrm{~nm}$ ), 11 fluorescence detectors and dispersed laser light detectors-forward scatter (FSC) and side scatter (SSC) were used for the analysis of Gal epitopes on the surface of examined cells. Optical alignment and functional stability tests were conducted with the CST system (Cytometer Setup and Tracking; Becton Dickinson). FACSFlow (Becton Dickinson) was used as the sheath fluid. The following cytometer configuration was applied: $85 \mu \mathrm{m}$ cell sorting nozzle and sheath fluid pressure of $45 \mathrm{psi}$. The characteristics of cells were obtained based on two non-fluorescent parameters, FSC and SSC, and one fluorescent parameter, red fluorescence (FL1 detector), from the AlexaFluor 647 fluorochrome, with detection obtained by the use of a 660/20 optical filter. Red laser $(633 \mathrm{~nm})$ was used for AlexaFluor 647 fluorochrome excitation. Cytometric analysis was conducted based on specific detector settings, which convert the collected signals into a logarithmical scale. The FSC detector was equipped with an optical filter of neutral density in order to weaken the dispersed light reaching it and enable conversion of the population of analysed cells on a two-dimensional scale to a graph showing the relations between the FSC and SSC. The 
threshold was set at the signal of the FSC detector. The data presented on the logarithmical scale were divided into four decades in the form of the signal surface from different detectors (FSC-A, SSC-A and FL1-A) and analysed with the use of FACS DIVA software (Becton Dickinson). The analysis of fluorescence signals preceded the doublets discrimination procedure with the use of height versus width scatter signals measurement, in order to discriminate single events (cells) from conglomerates. The populations were then defined by gating in the dot plots of red fluorescence (FL1) versus side scatter signals (SSC). Each sample was analysed four times. Determining the levels of Gal epitope on the surface of the analysed cells was conducted based on the median values of fluorescence signals from the FL1 detector in populations defined on a two-dimensional graph (FL1 and SSC).

\section{Human serum cytotoxicity test}

Having reached full confluence, the skin fibroblasts cultivated in vitro were rinsed with Hanks solution and trypsinised. The fibroblasts were collected by centrifugation and the debris was washed with Hanks solution and suspended in $200 \mu \mathrm{l}$ of one of three mediums: (A) $50 \%$ DMEM and $15 \%$ FBS in PBS, basic medium; (B) $50 \%$ HS (human serum) in PBS, test medium; (C) $50 \%$ thermally inactivated HS in PBS, control medium. The cells were incubated at $37^{\circ} \mathrm{C}$ for $30 \mathrm{~min}$. Then, $20 \mu \mathrm{l}$ of $0.4 \%$ trypan blue was added to $20 \mu \mathrm{l}$ of cell suspension and incubated for $5 \mathrm{~min}$ at room temperature. Living and dead cells were counted in Bürker's chamber with a volume of $0.9 \mathrm{~mm}^{3}$. Cells that were over or touching the top and left line of each square of $1 \mathrm{~mm}^{2}$ were counted, while the cells that were over or touching the bottom or right line were ignored. Each analysis was repeated 12 times. The percentage of living cells was calculated for each repetition. Then, the average numbers of living cells, dead cells and all cells from 12 repetitions were calculated.

\section{Results}

Obtaining transgenic pigs with $\alpha$-galactosidase expression

The pGal-GFPBsd gene construct was obtained by introducing cDNA coding for $\alpha$-galactosidase into a pTracer-EF/Bsd A plasmid. The correctness of the gene construct was confirmed by sequencing. The prepared pGal-GFPBsd gene construct (Fig. 1) was introduced based on the standard method of microinjection into one of the pronuclei of the zygote. 580 fertilised egg cells from 48 donors were subjected to microinjection. 529 zygotes were transplanted into the fallopian tubes of synchronised recipients (average of 33 zygotes/animal). From eight recipients, 37 piglets were obtained in the F0 generation, which were subjected to DNA analysis in order to detect the transgene by PCR. The analysis showed that one male piglet in the $\mathrm{F} 0$ generation (TG252) incorporated the transgene $(1 / 37 ; 2.7 \%$ ) (Fig. 2a). When the male TG252 reached puberty, its sperm was used for the artificial insemination (AI) of wild-type sows. As a result of AI, 75 piglets have been obtained so far, among which 12 were transgenic $(12 / 75 ; 16 \%)$. Changes in the size of the products analysed by PCR were not observed in transgenic pigs from the F1 generation, which means that the transgene is stably integrated with the genome and passed between consecutive generations (Fig. 2b). To confirm transgene integration, we conducted Southern analysis for all transgenic animals, which confirmed the presence of transgene in all of them. We show the results for TG252 (F0) and three representatives of the F1 generation, TG1183, TG1036 and TG1040, in Fig. 3. By using known concentrations of the transgene mixed with wild-type DNA, we were able to estimate the copy number in each of the animals. In the current analysis, the approximate number of transgene copies integrated was estimated as 16 .

From screening by Southern analysis, it can be determined that transgene is present, intact, in how many copies and what orientation. Neither of the other commonly used techniques yielded nearly the same amount of information. PCR analysis does not allow to quantify the copy number and, usually, can only confirm the presence of the transgene and cannot provide information as to either the intactness or orientation of the transgene. Despite its limitations, the technique is useful as a preliminary screening method. The use of PCR allows the identification of potential transgenic animals and reduces the number of samples for Southern analysis (Tinkle and Jay 2002).

\section{Cytogenetic analysis}

Karyotype evaluation of the transgenic animals was performed by the Giemsa method. The obtained pattern of bands on chromosomes was analysed and compared with the standard karyotype of domestic pig. The number, structure and architecture of chromosomes were unaffected in both the F0 (TG252) and F1 (TG1183) generations (Fig. 4a, b). In both generations (F0 and F1), the transgene was located only on chromosome 11, short arm, region 1, band 2. It was stable and integrated with genomic DNA (Fig. 4c, d). The transgene was integrated as concatemers of an estimated 16 copies on chromosome 11p12.

Flow cytometry

Flow cytometry was used for checking whether the transgene expression results in the presence of functional $\alpha$-galactosidase responsible for removing terminal residues of $\alpha$-D-galactose 
a
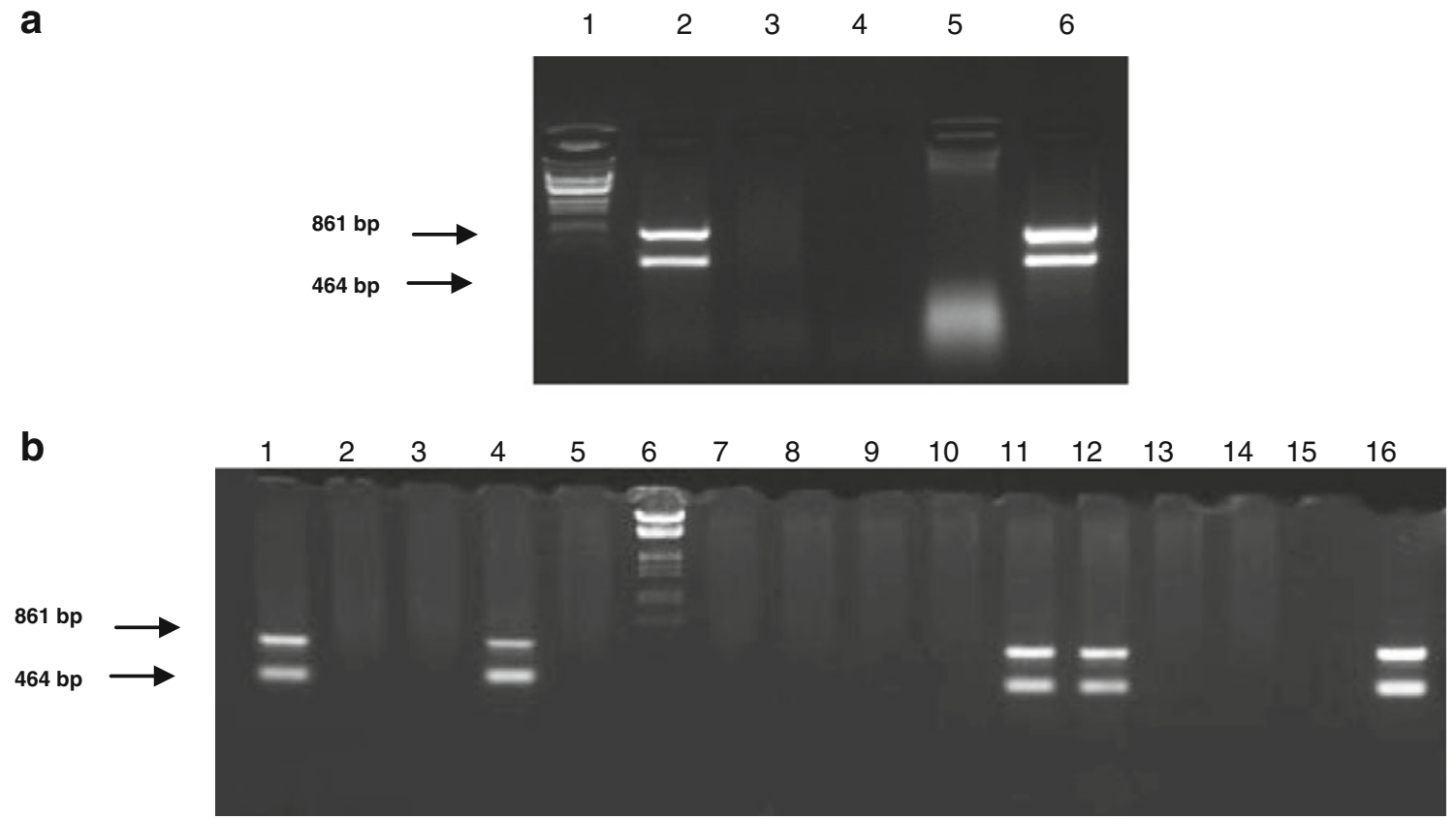

Fig. 2 Screening of the pGal-GFPBsd gene construct. Polymerase chain reaction (PCR) was used to amplify DNA fragments of $464 \mathrm{bp}$ and $861 \mathrm{bp}$. The separation of DNA fragments was conducted in $1.5 \%$ agarose gel. a Analysis of the integration with genomic DNA in pigs (F0). Line 1, size marker ( $\lambda$ DNA/HindIII, EcoRI); lane 2, DNA isolated from TG252; lane 3, DNA isolated from 253; lane 4, negative control

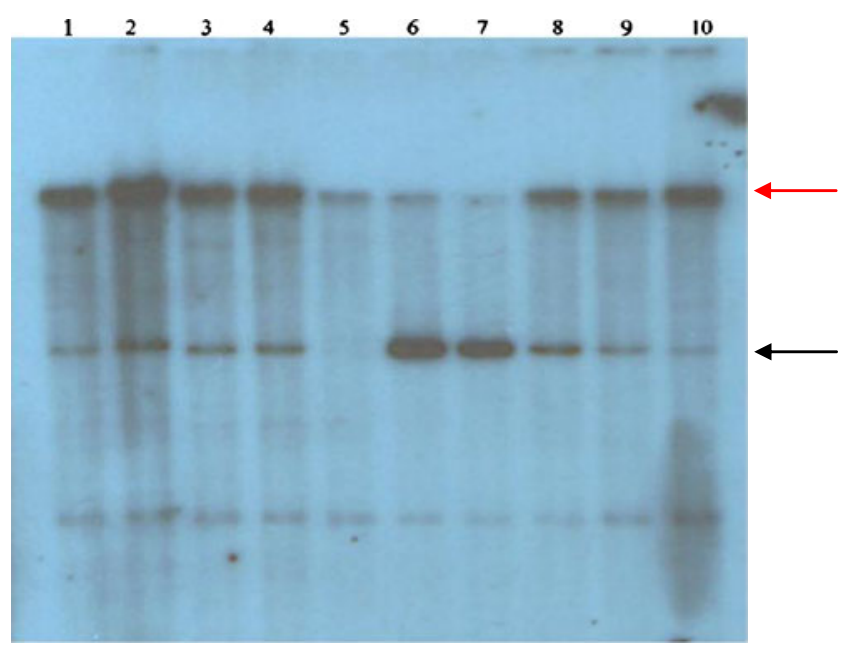

Fig. 3 Southern blot hybridisation analysis to confirm transgenic animals and estimate of copies number. DNA $(5.6 \mu \mathrm{g})$ isolated from transgenic animals TG252, TG1183, TG1036 and TG1040, respectively, carrying the pGAL-GFPBsd gene construct was digested with EcoRI and hybridised with a probe of $464 \mathrm{bp}$. The probe was a 32P-dATP-labelled PCR product of $464 \mathrm{bp}$ formed on plasmid DNA (pGAL-GFPBsd) with the use of the first pair of primers used for screening of the transgene. The probe detects a 7.3-kb band that represents the pGAL-GFPBsd genetic construct (black arrow). The red arrow probably indicates the band of non-specific hybridisation of the probe. Lanes 1-4 contain DNA samples from transgenic animals (TG252, TG1183, TG1036, TG1040); lane 5 wild-type pig DNA as a negative control; lanes 6-10 titration of the transgene mixed with digested wild-type genomic DNA representing $133.12,66.56,33.28,16.64$ and 8.32 copies, respectively (without DNA); lane 5, negative control (wild-type pig DNA); lane 6, positive control (pGal-GFPBsd gene construct). b Analysis of integration with genomic DNA in pigs (F1). Lanes 1-5 and 7-13, DNA isolated from potentially transgenic pigs; line 6, size marker ( $\lambda$ DNA/HindIII, EcoRI); line 14, negative control (wild-type pig DNA); line 15, negative control (without DNA); line 16, positive control (pGal-GFPBsd gene construct)

from the Gal $\alpha(1,3)$ Gal epitope, which, in consequence, should be connected with the reduction of the Gal antigen level on the surface of cells in pigs. Skin fibroblasts derived from wild-type animals, TG252 (F0) and TG1183 (F1), were used for the analysis. Before the analysis of $\alpha$-galactosidase functionality on the protein level carried out by measuring the amount of Gal antigen on the cell surface by flow cytometry, the enzyme was labelled by BS-IB4 lectin conjugated with AlexaFluor 647 fluorochorme. BS-IB4 lectin isolated from Griffonia simplicifolia (Bandeiraea simplicifolia) specifically binds Gal antigen and the fluorescent intensity of AlexaFluor 647 allows for the determination of the relative amount of labelled antigens on the surface of examined cells compared to nontransgenic cells. The median values of fluorescence signals from FL1 detector (AlexaFluor 647) are proportional to the Gal epitope levels on the surface of the analysed cells. Staining with BS-IB4 lectin showed a significant reduction of fluorescence intensity in the case of fibroblasts from both the founder from the F0 generation (TG252) as well as the transgenic animal from the $\mathrm{F} 1$ generation compared to control fibroblasts of wild-type animals. From the obtained results, it can be concluded that the transgene integrated with genomic DNA of pigs is fully functional, was incorporated into an active transcription region and is expressed.

The analysis showed decreased levels of Gal antigen in transgenic animals in F0 and F1 compared to wild-type animals. The reduction of Gal epitope expression was $58.21 \%$, 
a
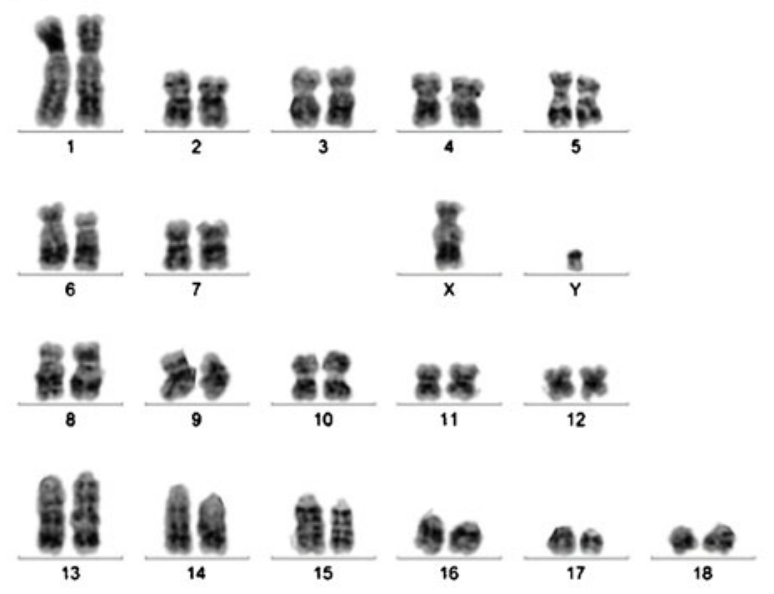

C

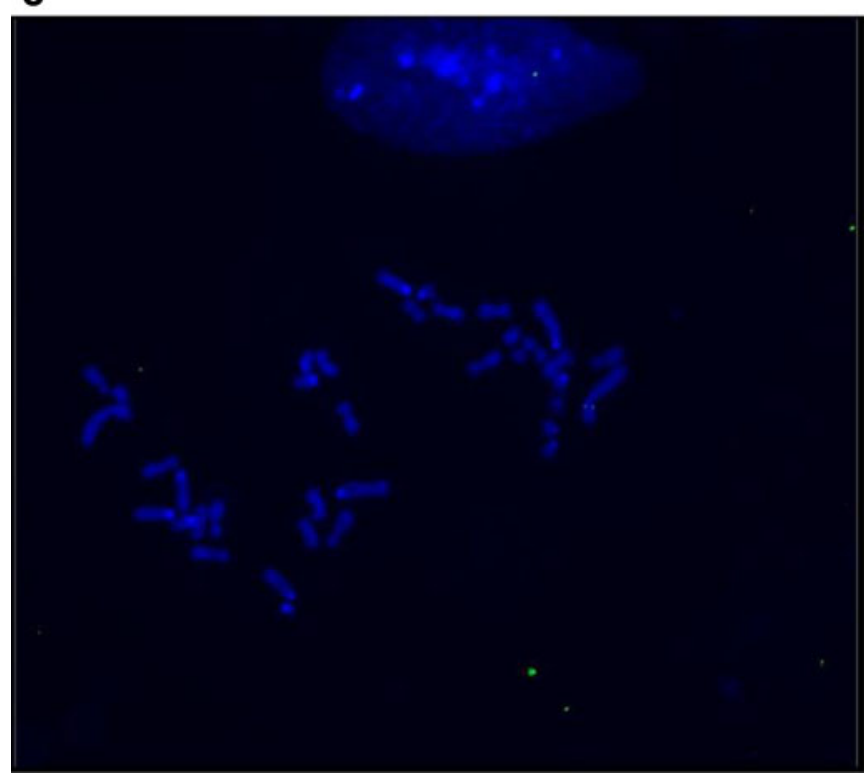

Fig. 4 Cytogenetic analysis of animals expressing human $\alpha$-galactosidase. a Correct karyotype of a pig from the F0 generation, TG252 (38, XY). b Correct karyotype of a pig from the F1 generation, TG1183 (38, $\mathrm{XX}$ ). c Post-hybridisation image of metaphase chromosomes from the interphase nucleus of a cell line derived from skin fibroblasts of TG252.

$59.67 \%, 56.67 \%$ and $65.09 \%$. The level of Gal epitope expression observed in F1 animals was similar to the F0 animal (TG252). The fluorescence intensity decrease in TG252 was $2.59 \times$ in relation to the wild-type control, while for the pig from F1 (in this study TG1183 and, additionally, TG1036 and TG1040) was 2.86-2.31× (Fig. 5b). Additionally, fibroblasts from cell lines derived from the TG1154 transgenic pig with the expression of $\alpha 1,2$-fucosyltransferase, obtained by the study group in the previous years (Lipiński et al. 2010), were used for comparative analyses. For the transgenic pig TG1154, a relative decrease of more than two and a half $(62.8 \%)$ in the amount of Gal antigen on the cell surface was observed in relation to the wild-type control, measured b

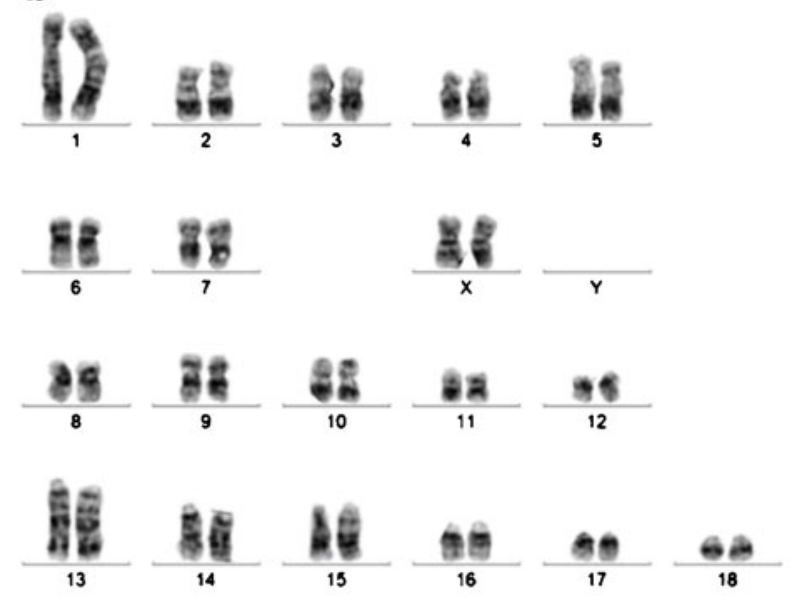

d

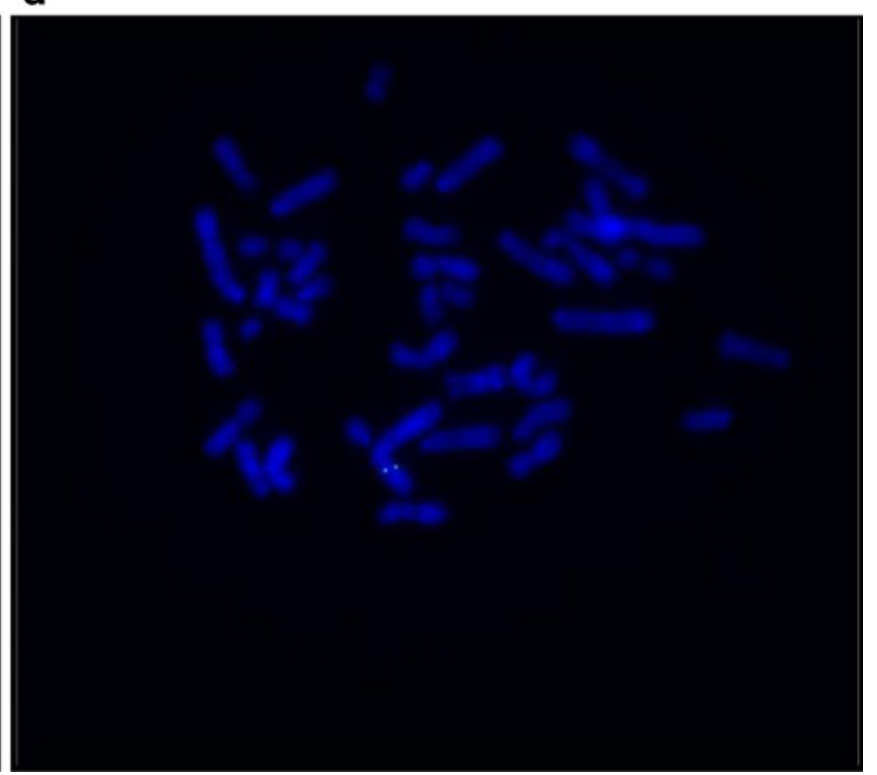

Signal on chromosome 11q12. FITC-labelled marker, chromosomes and nucleus stained by DAPI. d Post-hybridisation image of metaphase chromosomes from a cell line derived from skin fibroblasts of the F1 pig. Signal on chromosome 11q12. FITC-labelled marker, chromosomes stained by DAPI

by median values of fluorescence intensity after staining with lectin BS-IB4 (Fig. 5a).

\section{Human serum cytotoxicity test}

Another test verifying the pGal-GFPBsd transgene functionality was the survival rate analysis of skin fibroblasts derived from TG252 (F0) and TG1183 (F1) in comparison to the survival rate of other wild-type cell lines in the presence of human serum acting as a source of complement system elements. Activation of the complement system results in a number of reactions which effect the formation of a complex attacking the membrane. It is responsible for forming channels 


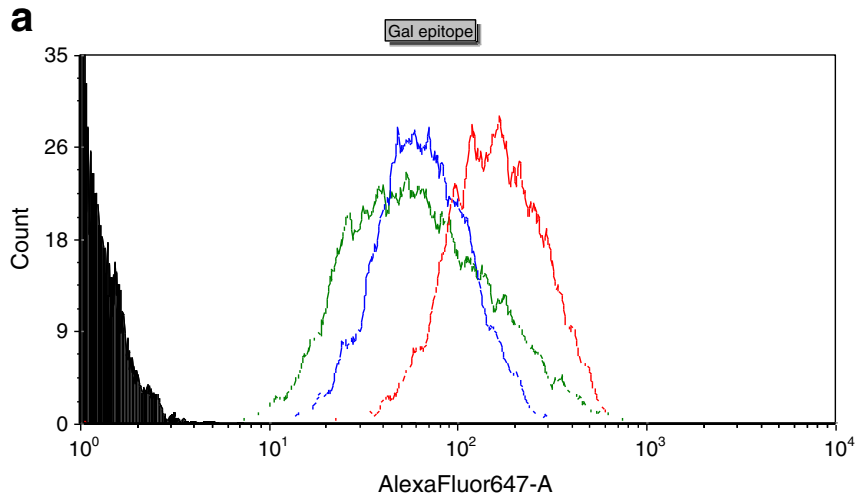

Fig. 5 Transgene expression analysis by flow cytometry. Fibroblasts derived from the following animals were analysed: wild-type, pig TG252 (F0), transgenic pigs F1 (TG1183, TG1036, TG1040), TG1154 expressing 1,2-fucosyltransferase labelled by BS-IB4 lectin (detects Gal epitope), conjugated with AlexaFluor 647 fluorochrome. a The $x$-axis shows the fluorescence intensity, the $y$-axis shows the number of cells which were not labelled (black lines), labelled wild-type cells (red line), transgenic animal TG252 (blue line) and transgenic animal TG1154 (green line). The analysis showed decreased levels of $\mathrm{Gal}$ antigen in transgenic animals compared to wild-type animals. The fluorescence fell by $58.9 \%$ for the pig with $\alpha$-galactosidase (TG252) expression and by $62.8 \%$ for the

in the cellular membrane that deform its structures through changing lipid orientation and releasing liposomes. As a result of the activity of the membrane-attacking complex, destruction of the recognised cells takes place. Control medium contained $50 \%$ of inactivated human serum where the components of the human complement system should not be active. This medium was used as the toxicity control of compounds not belonging to the complement system which were present in the human serum. The survival rate analysis showed that there is no statistically significant difference between the survival rate of cells in the basic medium (A) and in the control medium (C), so it is not the source of toxic compounds. The survival rate was determined by the incubation of cell suspension with a solution of trypan blue, a dye which penetrates the structure of dead cells whose membrane is fragmented.

The analyses conducted for the test medium, where living cells constituted only $22.05 \%(+/-3.65 \%)$, showed that the compounds present in the serum affected adversely the survival rate of wild-type pigs compared to basic medium $(89.87 \%,+/-6.09 \%)$, with no elements of the human complement system. Similar comparative analysis conducted for a line of fibroblasts derived from a transgenic animal (TG252) expressing $\alpha$-galactosidase revealed a minor decrease of the cell survival rate in test medium $(91.67 \%,+/-3.13 \%)$ in relation to the basic medium $(98.53 \%,+/-0.06 \%)$. Also, the cells derived from TG1183 (F1) were characterised by high resistance to elements of the human complement system present in human serum. The survival rate was $97.23 \%(+/-0.93 \%)$ in the basic medium without human serum and $93.37 \%(+/-2.15 \%)$ in the test medium. A similar survival rate analysis was conducted for b

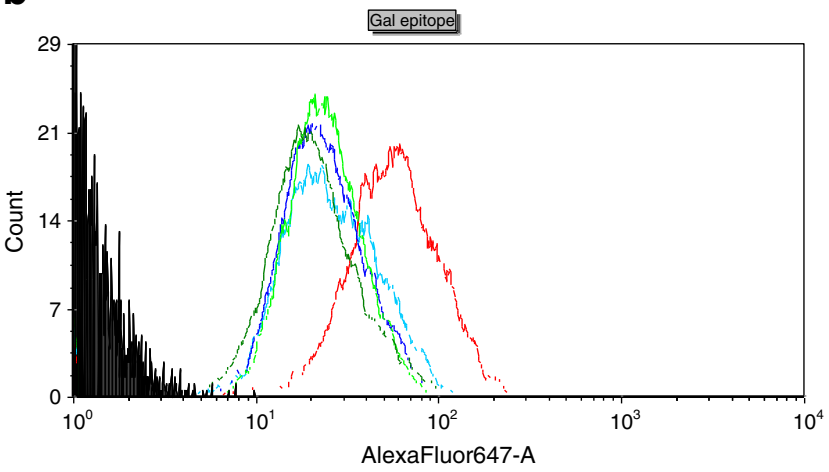

pig with $\alpha 1,2$-fucosyltransferase (TG1154) expression compared to control. b The $x$-axis shows the fluorescence intensity, the $y$-axis shows the number of cells which were not labelled (black lines), labelled wild-type cells (red line), transgenic animal TG252 (F0) (light green line) and transgenic animal TG1183 (F1) (dark blue line), transgenic animal TG1036 (F1) (light blue line) and transgenic animal TG1040 (F1) (dark green line). The analysis showed decreased levels of Gal antigen in transgenic animals in F0 and F1 compared to wild-type animals. The reduction of $\mathrm{Gal}$ epitope expression was, respectively, $58.21 \%$, $59.67 \%, 56.67 \%$ and $65.09 \%$. The level of Gal epitope expression observed in the F1 animals was similar to the F0 animal (TG252)

a cell line derived from transgenic animal TG1154 expressing $\alpha 1,2$-fucosyltransferase. The presence of transgene protected the skin fibroblasts from the complement system activity and the percentage loss of living cells was not significant. The survival rate measured by an average number of living cells compared to the total number of counted cells in 12 repetitions amounted to $97.92 \%(+/-1.17 \%)$ in the basic medium and $92.35 \%(+/-2.82 \%)$ in the test medium (Fig. 6).

\section{Discussion}

One of the big challenges in xenotransplantation is that one barrier needs to be totally resolved before the next is fully unmasked. The complexity of processes responsible for initiating immunological reaction does not allow a onedimensional approach to xenotransplantation. Several strategies need to be combined for obtaining an additive effect in one transgenic organism in order to increase the chance of stopping the immunological aggression against the cells of the xenograft and its consequent survival. Modern genetic engineering is facing a serious problem of producing, in the shortest possible time, polytransgenic animals by interbreeding monotransgenic animals or simultaneous transfection with several transgenes in mono- or polycistronic transgene expression. In the case of xenotransplantation, the opportunity to develop and incorporate multiple genetic modifications simultaneously will be limited until each mechanism is identified. It was proved that, in the case of coinjection of two similar gene constructs, the expression of both transgenes shows 


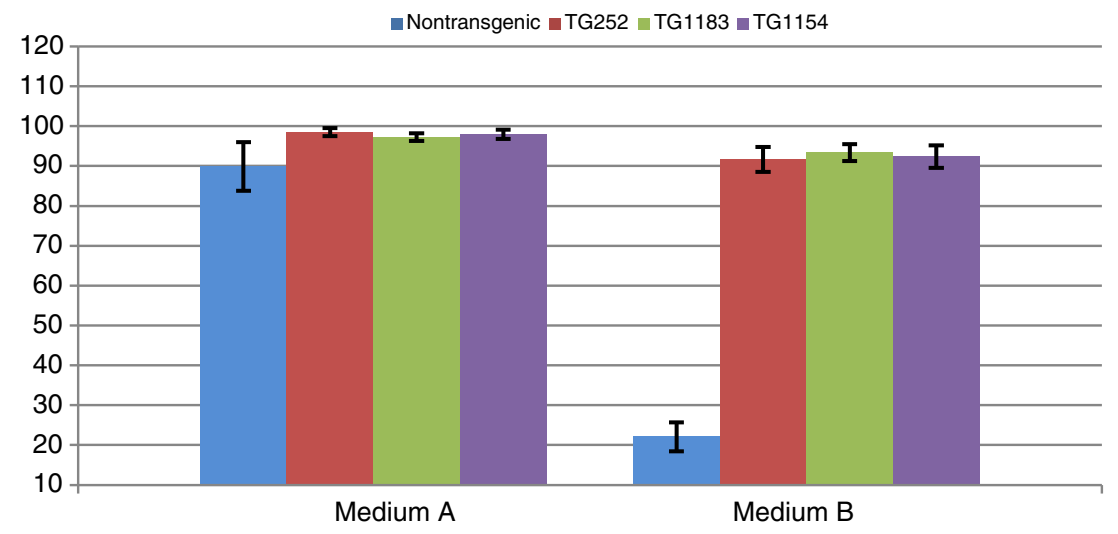

Fig. 6 Survival rate analysis with standard deviations for cells from wild-type animal (blue), from F0 (TG252) with $\alpha$-galactosidase expression (red), F1 animal (TG1183) with $\alpha$-galactosidase expression (green) and TG1154 with the expression of $\alpha 1,2$-fucosyltransferase (purple)

effectiveness similar to that observed in the case of single transgenes introduced into two different animals (Jankowsky et al. 2001; Goldman et al. 2004).

Hyperacute rejection of xenografts is the basic immunological limitation that makes transplanting animal organs in humans unsuccessful. Experimental data and some clinical experience show that there are three main directions that can be followed: depletion of anti-pig antibodies by the development of homozygous pigs for a1,3-galactosyltransferase gene-knockout (GTKO), enzymatic removal of Gala1,3-Gal epitope or complement inhibition, or some combination of these approaches (Pierson 2009). As we started our experiments in 2002, there were several concerns about pigs with the GTKO phenotype. First is the possible indispensability of Gal in pigs and its essentiality for cellular function. Second is the presence of another galactosyltransferase which is capable of producing the Gal xenoantigen on glycolipids. And, finally, the deletion of the Gala1,3-Gal epitope would expose new, potentially immunogenic antigens (Gock et al. 2011). In the human organism, even $90 \%$ xenoreactive antibodies recognise the Gal $\alpha(1,3) \mathrm{Gal}$ epitope, but there is still the remaining $10 \%$ to be challenged. As a consequence, we chose two strategies involving molecular modifications of the animal genomes aiming at preventing the hyperacute rejection from the immunological system. The first strategy described in this study helps to prevent HAR by means of a reduction of the epitope through the introduction and expression of a sequence coding for $\alpha$-galactosidase, which eliminates the effect of $\alpha 1,3$-galactosyltransferase as a result of removing the terminal $\alpha$-D-galactose in the Gal $\alpha(1,3)$ Gal structure. Microinjection allowed us to obtain a transgenic pig with an integrated, functional and stable pGal-GFPBsd transgene. Both the transgenic hog TG252 as well as animals in the F1 generation were characterised by a GLA expression level ensuring the derived lines of fibroblast protection from the destructive human complement system in the cytotoxicity test. The survival rate measured in the basic medium and in test medium B containing $50 \%$ human serum. The survival rate was measured by an average number of living cells compared to the total number of counted cells in 12 repetitions. The percentage cell survival rate is shown on the $y$-axis

in the medium containing human serum decreased by $7 \%$ at most, which suggests decreased reactivity in relation to transgenic pig cells. The observed decrease of the cell survival rate may result from the fact that GLA, when removing the terminal galactose from the surface of the Gal $\alpha(1,3)$ Gal structure, exposes the unmodified residue of $\mathrm{N}$-acetyllactosamine, which may be a potential cryptoantigen causing immunological response, irrespective of XNA (Watier et al. 1996). Flow cytometry revealed a significant reduction of Gal antigen on the surface of fibroblasts compared to the amount on the cell surface of wild-type animals.

In previous studies, we focused on attempting to decrease the level of Gal antigen synthesis on the surface of cells by replacing it with a commonly accepted $\mathrm{H}$ antigen ( 0 antigen of the $\mathrm{ABO}$ blood group system) by means of the expression of functional human enzyme $\alpha 1,2 \mathrm{FT}$ in transgenic pigs (Jura et al. 2004; Lipiński et al. 2010). Pigs carry an endogenous gene coding for $\alpha 1,2$-fucosyltransferase; however, the $\mathrm{H}$ epitope produced by this enzyme is not expressed on the endothelial surface of blood vessels (Oriol et al. 1993; Sharma et al. 1996). A high level of reduction in the synthesis of the Gala $(1,3) \mathrm{Gal}$ epitope observed in vivo on the surface of the endothelium is, hence, an effect of artificially generated competition between the endogenous $\alpha 1,3 \mathrm{GT}$ and $\alpha 1,2 \mathrm{FT}$ introduced experimentally. The analysis we conducted showed a significant reduction in Gal antigen on the surface of fibroblasts derived from transgenic animals, with additional copies of the $\alpha 1,2 \mathrm{FT}$ gene introduced to their genomes compared to the number of epitopes occurring on the surface of cells in wild-type animals. The reduction of Gal antigen on the cell surface in transgenic animals is, hence, a result of $\mathrm{H}$ transferees, which competes with 1,3-galactosyltransferase for the acceptor molecule. It is precisely correlated with the observed, increased levels of $\mathrm{H}$ antigen (Jura et al. 2004; Lipiński et al. 2010).

We plan to interbreed the described transgenic animals and obtain the simultaneous expression of $\alpha 1,2$-fucosyltransferase 
and $\alpha$-galactosidase. Through the combination of two modifications, we will try to obtain the cumulative effect of decreasing the level of Gala $(1,3) \mathrm{Gal}$ epitope. The research to date confirmed that GLA expression decreased the level of Gal $\alpha(1,3) \mathrm{Gal}$ epitope by $78 \%$, and the coexpression of $\alpha$ galactosidase and 1,2-fucosyltransferase eliminated it entirely from the surface of SV40-immortalised aortic porcine endothelial cells (PEDSV.15) (Jia et al. 2004). The enzyme $\alpha 1,2 \mathrm{FT}$ located within the medial compartment of the Golgi apparatus cisterns shows its activity earlier than the trans endogenous $\alpha 1,3 \mathrm{GT}$ present in the trans compartment. The maturing oligosaccharide, when migrating through the Golgi apparatus from cis to trans compartments, cannot accept the terminal galactose in a reaction catalysed by $\alpha 1,3 \mathrm{GT}$ because of the previous fucosylation of $\mathrm{N}$-acetyllactosamine resulting from $\alpha 1,2 \mathrm{FT}$ activity (Hartel-Schenk et al. 1991). The simultaneous transformation of COS cells with $\alpha 1,2 \mathrm{FT}$ and $\alpha 1,3 \mathrm{GT}$ coding genes showed the shift of the expression profile towards the production of H epitope (Sandrin et al. 1995). The expression of $\alpha 1,2 \mathrm{FT}$ does not result in total Gal $\alpha(1,3) \mathrm{Gal}$ epitope elimination, so introducing additional copies of genes coding for $\alpha 1,3 \mathrm{GT}$ would supplement the activity of 1,2-fucosyltransferase. Introducing gene coding for $\alpha$-galactosidase removing the terminal $\alpha$-D-galactose should result in a further reduction of the amount of functional Gal $\alpha(1,3) \mathrm{Gal}$ epitope formed as a result of competition between $\alpha 1,3 \mathrm{GT}$ and $\alpha 1,2 \mathrm{FT}$. Given that the effect of GLA activity, when a solution of this enzyme is administered, is transient, the integration of several strategies preventing HAR is even more advisable. Regardless of the initial reduction in the binding of IgM antibodies, after ex vivo perfusion of pig kidneys with endo- $\beta$-galactosidase $C$ solution, the resynthesis of Gal $\alpha(1,3) \mathrm{Gal}$ epitope on the cell surface and increased XNA reactivity are observed. Obtaining a proper therapeutic effect would be linked to the necessity of constant supplementation of the recipient with GLA solution (Ogawa et al. 2000). More recently, Alexion Pharmaceuticals produced a combined GTKO with H-transferase transgenic pig to "compete out" any potential antigen-producing transferases, including the putative secondary galactosyltransferase (Ramsoondar et al. 2003).

Other strategies of preventing hyperacute rejection of xenotransplants conducted in our team involve enzymatic regulation of the complement system, which concerns blocking the formation of membrane-attacking complex, and inactivation of the $\alpha 1,3 \mathrm{GT}$ gene responsible for the final Gal $\alpha(1,3) \mathrm{Gal}$ structure (unpublished data). The existence of genetic modifications ensuring the expression of possibly the highest number of regulators of the complement system and altering the distribution of sugars on the surface of pig cells is quite likely to contribute to both quantitatively and qualitatively limiting the use of pharmacological treatment, which weakens the immunological system by blocking its functions. Only animals with numerous genetic modifications obtained through the interbreeding of organisms with single modifications or through cotransfection with several gene constructs will become a reservoir of organs for medical purposes (Cooper 2012).

Acknowledgments This work was supported by project no. N R12 003606.

Open Access This article is distributed under the terms of the Creative Commons Attribution License which permits any use, distribution, and reproduction in any medium, provided the original author(s) and the source are credited.

\section{References}

Chomczynski P, Sacchi N (1987) Single-step method of RNA isolation by acid guanidinium thiocyanate-phenol-chloroform extraction. Anal Biochem 162(1):156-159

Cooper DK (2012) A brief history of cross-species organ transplantation. Proc (Bayl Univ Med Cent) 25(1):49-57

Galili U, Swanson K (1991) Gene sequences suggest inactivation of alpha-1,3-galactosyltransferase in catarrhines after the divergence of apes from monkeys. Proc Natl Acad Sci U S A 88(16):7401-7404

Galili U, Shohet SB, Kobrin E, Stults CL, Macher BA (1988) Man, apes, and Old World monkeys differ from other mammals in the expression of alpha-galactosyl epitopes on nucleated cells. J Biol Chem 263(33):17755-17762

Gock H, Nottle M, Lew AM, d'Apice AJ, Cowan P (2011) Genetic modification of pigs for solid organ xenotransplantation. Transplant Rev (Orlando) 25:9-20

Goldman IL, Kadulin SG, Razin SV (2004) Transgenic animals in medicine: integration and expression of foreign genes, theoretical and applied aspects. Med Sci Monit 10(11):RA274-RA285

Gustavsson I (1988) Standard karyotype of the domestic pig. Committee for the Standardized Karyotype of the Domestic Pig. Hereditas 109(2):151-157

Hartel-Schenk S, Minnifield N, Reutter W, Hanski C, Bauer C, Morré DJ (1991) Distribution of glycosyltransferases among Golgi apparatus subfractions from liver and hepatomas of the rat. Biochim Biophys Acta 1115(2):108-122

Jankowsky JL, Slunt HH, Ratovitski T, Jenkins NA, Copeland NG, Borchelt DR (2001) Co-expression of multiple transgenes in mouse CNS: a comparison of strategies. Biomol Eng 17(6):157-165

Jia Y, Ren H, Gao X, Ji S, Yang J, Liu Z, Li S, Zhang Y (2004) Expression of human alpha-galactosidase and alpha1,2-fucosyltransferase genes modifies the cell surface Galalpha1,3Gal antigen and confers resistance to human serum-mediated cytolysis. Chin Med Sci J 19:31-37

Jura J, Słomski R, Smorag Z, Gajda B, Wieczorek J, Lipiński D, Kalak R, Juzwa W, Zeyland J (2004) Production of transgenic pigs suitable for xenotransplantation with the use of standard DNA microinjection. Ann Anim Sci 4:321-327

Larsen RD, Ernst LK, Nair RP, Lowe JB (1990) Molecular cloning, sequence, and expression of a human GDP-L-fucose:beta-Dgalactoside 2-alpha-L-fucosyltransferase cDNA that can form the $\mathrm{H}$ blood group antigen. Proc Natl Acad Sci U S A 87:6674-6678

LaVecchio JA, Dunne AD, Edge AS (1995) Enzymatic removal of alpha-galactosyl epitopes from porcine endothelial cells diminishes the cytotoxic effect of natural antibodies. Transplantation 60(8):841-847

Lipiński D, Jura J, Zeyland J, Juzwa W, Mały E, Kalak R, Bochenek M, Plawski A, Szalata M, Smorag Z, Słomski R (2010) Production of transgenic pigs expressing human $\alpha 1,2$-fucosyltransferase to avoid humoral xenograft rejection. Med Wet 66(5):316-322 
Luo Y, Wen J, Luo C, Cummings RD, Cooper DK (1999) Pig xenogeneic antigen modification with green coffee bean alpha-galactosidase. Xenotransplantation 6(4):238-248

McMorrow IM, Comrack CA, Sachs DH, DerSimonian H (1997) Heterogeneity of human anti-pig natural antibodies cross-reactive with the Gal(alpha1,3)Galactose epitope. Transplantation 64(3):501510,15

Ogawa H, Muramatsu H, Kobayashi T, Morozumi K, Yokoyama I, Kurosawa N, Nakao A, Muramatsu T (2000) Molecular cloning of endo-beta -galactosidase $\mathrm{C}$ and its application in removing alpha galactosyl xenoantigen from blood vessels in the pig kidney. J Biol Chem 275(25):19368-19374

Organ Procurement and Transplantation Network (OPTN) (2011) Home page at: http://optn.transplant.hrsa.gov/

Oriol R, Ye Y, Koren E, Cooper DK (1993) Carbohydrate antigens of pig tissues reacting with human natural antibodies as potential targets for hyperacute vascular rejection in pig-to-man organ xenotransplantation. Transplantation 56(6):1433-1442

Osman N, McKenzie IF, Ostenried K, Ioannou YA, Desnick RJ, Sandrin MS (1997) Combined transgenic expression of alphagalactosidase and alpha1,2-fucosyltransferase leads to optimal reduction in the major xenoepitope Galalpha(1,3)Gal. Proc Natl Acad Sci U S A 94:14677-14682

Pierson RN 3rd (2009) Antibody-mediated xenograft injury: mechanisms and protective strategies. Transpl Immunol 21(2):65-69

Ramsoondar JJ, Macháty Z, Costa C, Williams BL, Fodor WL, Bondioli KR (2003) Production of alpha 1,3-galactosyltransferase-knockout cloned pigs expressing human alpha 1,2-fucosylosyltransferase. Biol Reprod 69:437-445
Sandrin MS, McKenzie IF (1994) Gal alpha $(1,3) \mathrm{Gal}$, the major xenoantigen(s) recognised in pigs by human natural antibodies. Immunol Rev 141:169-190

Sandrin MS, Vaughan HA, Dabkowski PL, McKenzie IF (1993) Antipig IgM antibodies in human serum react predominantly with Gal(alpha 1-3)Gal epitopes. Proc Natl Acad Sci U S A 90:1139111395

Sandrin MS, Fodor WL, Mouhtouris E, Osman N, Cohney S, Rollins SA, Guilmette ER, Setter E, Squinto SP, McKenzie IF (1995) Enzymatic remodelling of the carbohydrate surface of a xenogenic cell substantially reduces human antibody binding and complement-mediated cytolysis. Nat Med 1(12):1261-1267

Sharma A, Okabe J, Birch P, McClellan SB, Martin MJ, Platt JL, Logan JS (1996) Reduction in the level of Gal(alpha1,3)Gal in transgenic mice and pigs by the expression of an alpha(1,2)fucosyltransferase. Proc Natl Acad Sci U S A 93(14):7190-7195, 9

Tinkle BT, Jay G (2002) Molecular approaches involved in mammalian gene transfer: analysis of transgene integration. In: Pinkert CA (ed) Transgenic animal technology: a laboratory handbook, 2nd edn. Academic Press, San Diego

Watier H, Guillaumin JM, Piller F, Lacord M, Thibault G, Lebranchu Y, Monsigny M, Bardos P (1996) Removal of terminal alphagalactosyl residues from xenogeneic porcine endothelial cells. Decrease in complement-mediated cytotoxicity but persistence of IgG1-mediated antibody-dependent cell-mediated cytotoxicity. Transplantation 62(1):105-113

Yan JL, Yu LY, Zhu LH, Guo LH (2003) Expression of human alphagalactosidase leads to reduction of major xenoepitope Galalpha(1,3) Gal in NIH 3 T3 cell. Acta Pharmacol Sin 24(10):985-990 